ACCEPTED MANUSCRIPT

\title{
Generating strong room-temperature photoluminescence in black phosphorus using organic molecules
}

To cite this article before publication: Sruthi Kuriakose et al 2018 2D Mater. in press https://doi.org/10.1088/2053-1583/aae869

\section{Manuscript version: Accepted Manuscript}

Accepted Manuscript is "the version of the article accepted for publication including all changes made as a result of the peer review process, and which may also include the addition to the article by IOP Publishing of a header, an article ID, a cover sheet and/or an 'Accepted

Manuscript' watermark, but excluding any other editing, typesetting or other changes made by IOP Publishing and/or its licensors"

This Accepted Manuscript is @ 2018 IOP Publishing Ltd.

During the embargo period (the 12 month period from the publication of the Version of Record of this article), the Accepted Manuscript is fully protected by copyright and cannot be reused or reposted elsewhere.

As the Version of Record of this article is going to be / has been published on a subscription basis, this Accepted Manuscript is available for reuse under a CC BY-NC-ND 3.0 licence after the 12 month embargo period.

After the embargo period, everyone is permitted to use copy and redistribute this article for non-commercial purposes only, provided that they adhere to all the terms of the licence https://creativecommons.org/licences/by-nc-nd/3.0

Although reasonable endeavours have been taken to obtain all necessary permissions from third parties to include their copyrighted content within this article, their full citation and copyright line may not be present in this Accepted Manuscript version. Before using any content from this article, please refer to the Version of Record on IOPscience once published for full citation and copyright details, as permissions will likely be required. All third party content is fully copyright protected, unless specifically stated otherwise in the figure caption in the Version of Record.

View the article online for updates and enhancements. 


\section{Generating strong room-temperature} photoluminescence in black phosphorus using organic molecules

Sruthi Kuriakose, ${ }^{1}$ Taimur Ahmed, ${ }^{1}$ Patrick D. Taylor, ${ }^{2}$ Yi Zhu, ${ }^{4}$ Michelle J.S. Spencer, ${ }^{2}$ Sivacarendran Balendhran, ${ }^{1}$ Yuerui Lu, ${ }^{4}$ Vipul Bansal, ${ }^{* 3}$ Sharath Sriram, ${ }^{1}$ Madhu Bhaskaran ${ }^{1}$ and Sumeet Walia*1

${ }^{1}$ Functional Materials and Microsystems Research Group and Micro Nano Research Facility, School of Engineering, RMIT University, Melbourne VIC 3001, Australia

${ }^{2}$ School of Science, RMIT University Melbourne, 3001 Victoria, Australia

${ }^{3}$ Ian Potter NanoBioSensing Facility and NanoBiotechnology Research Laboratory, School of Science, RMIT University, Melbourne VIC 3001, Australia

${ }^{4}$ Research School of Engineering, College of Engineering and Computer Science, Australian National University, Canberra, ACT 2601, Australia

E-mail: sumeet.walia@rmit.edu.au,vipul.bansal@rmit.edu.au

Received xxxxxx

Accepted for publication $\operatorname{xxxxx}$

Published xxxxxx

\section{Abstract}

Black phosphorus (BP) exhibits fascinating thickness dependent optical and electronic characteristics. However, photoluminescence (PL) emission in the visible spectrum does not exist for multi-layer BP and requires the achievement of single layer, which are highly environmentally sensitive. This poses significant challenges in realizing the true potential of BP as multi-layer BP exhibits exciting optical properties for a range of applications. Here, for the first time we reveal visible range room-temperature photoluminescence (PL) in multi-layered black phosphorus (BP) via chemical doping using organic molecules. We find the drastic enhancement of PL originates from the adsorption of p-type dopants and offer further insight using Density Functional Theory (DFT) calculations. The reported non-destructive method creates a pathway to precisely control optical and electronic properties thereby expanding the application horizon for multilayer BP that is environmentally robust compared to monolayer.

Keywords: Black phosphorus, Charge transfer, Doping, Multi-layer, Photoluminescence

\section{Introduction}

Black phosphorus (BP), an elemental analogue of graphene, is the most stable allotrope of phosphorus. It has recently attracted a great deal of attention from the viewpoints of fundamental physics as well as a plethora of applications. ${ }^{[1-6]}$ This material has earned its place among the family of twodimensional (2D) semiconductor materials owing to its wideranging thickness-dependent properties, ${ }^{[3,7,8]}$ which make it a promising layered material for application in photo detectors, ${ }^{[4,9,10]}$ photovoltaic cells, ${ }^{[11,12]}$ and bio and gas sensors ${ }^{[13-15]}$ amongst many others.

$\mathrm{BP}$ is particularly suitable for optoelectronic applications, as it possesses a tunable layer-dependent direct band gap. It is responsive to a wide range of optical wavelengths, beyond the spectral region covered by the more common semiconducting two-dimensional (2D) transition metal dichalcogenides. ${ }^{[16-18]}$ Since BP is a direct bandgap material, which varies in value depending on the number of fundamental layers, it exhibits PL emission peaks that are highly dependent on thickness. ${ }^{[19]}$ While thick BP flakes exhibit PL in the infrared range of the light spectrum, ${ }^{[20,21]}$ a thickness-dependent blue-shift allows monolayer BP (phosphorene) to exhibit PL emission in the visible/ near-infrared range. ${ }^{[7,12,19,22]}$ The control over BP thickness $^{[23]}$ represents the commonly explored pathway to modulate its optical properties. However, thin BP flakes are prone to rapid ambient degradation and introduction of defects if thinning processes are deployed. ${ }^{[19,24-27]}$ Therefore, a highly desirable feature would be to achieve PL emission 
from thicker BP multilayers at visible wavelengths. This will eliminate the need to obtain stable BP monolayers and enable incorporation of the more robust multilayer BP in device applications. Furthermore, multi-layer BP exhibits exciting properties at infrared and telecommunications wavelengths.

Chemical doping is known to be an easy and effective pathway to modulate the carrier density of a variety of $2 \mathrm{D}$ material systems and other nanostructures. ${ }^{[8,28-36]}$ If dopant molecules are carefully chosen based on their redox potentials, they are capable of generating shifts in the Fermi levels of 2D material systems that can ensure the tunability of optical and electrical properties of the material.
In this paper, we demonstrate the emergence of highly intense PL at room temperature in the visible region of the electromagnetic spectrum in multilayer BP using a solutionbased chemical doping technique. It is shown that relative to the chemical potential of BP, the $p$-type dopants result in the appearance of highly intense PL, whereas the $n$-type dopant does not result in any such emergence or enhancements in PL. The presented study therefore offers a pathway to engineer a PL response via chemical doping that can be controlled based on the redox potential of the dopant relative to BP. This provides a new opportunity to expand the application base of multi layered BP. a

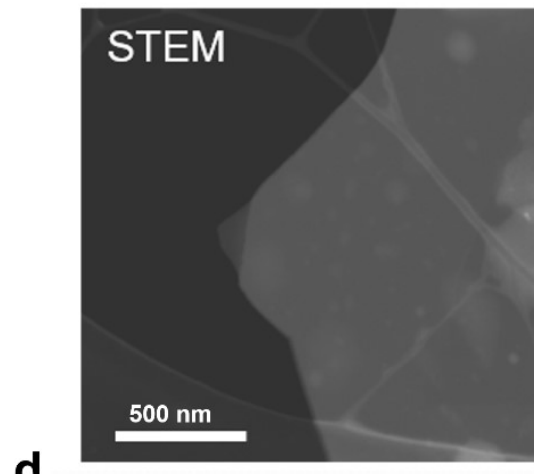

d

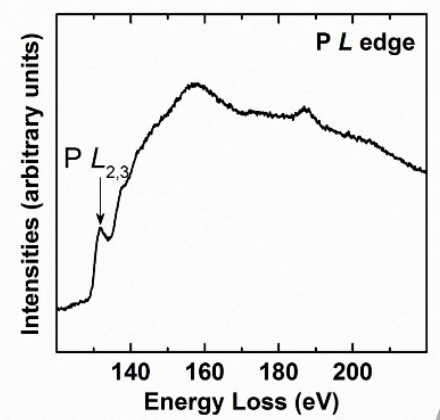

b

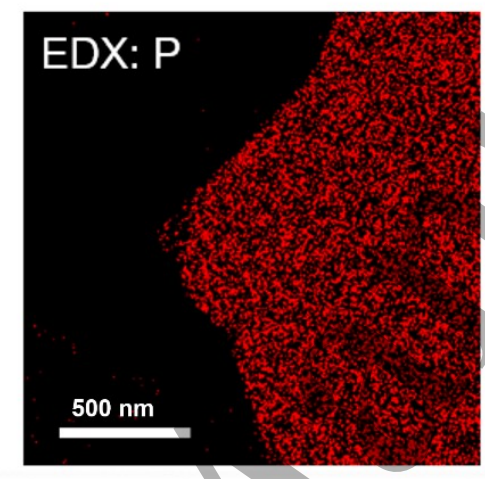

e

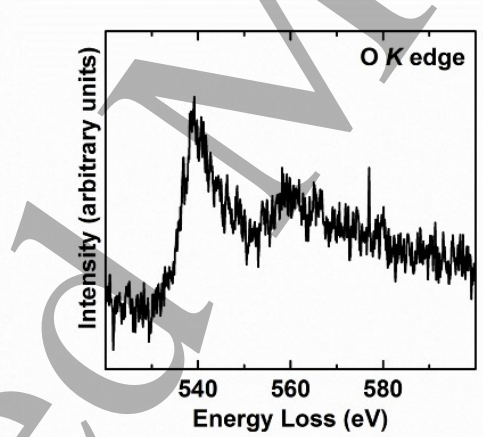

C

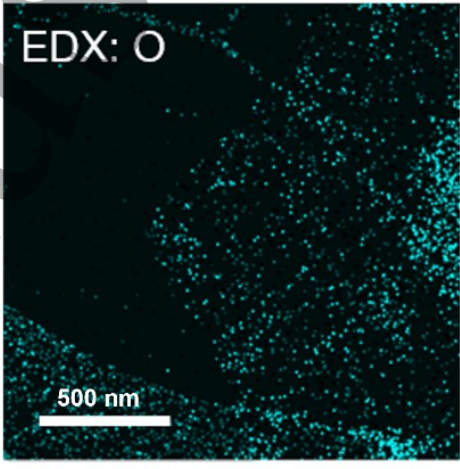

f

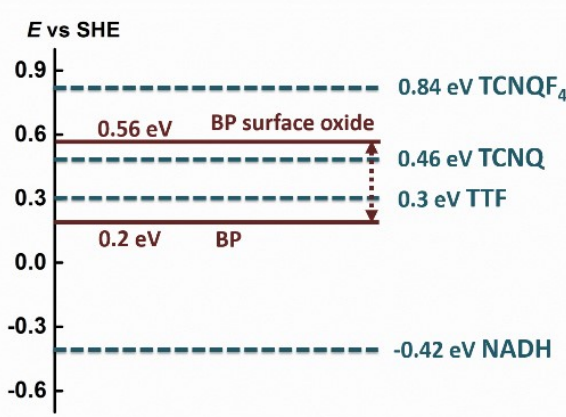

Figure 1: (a) Scanning transmission electron micrograph of a BP flake exfoliated on a carbon grid. Energy dispersive X-ray spectroscopy elemental maps of (b) phosphorus and (c) oxygen corresponding to the BP flake in (a). The electron energy loss spectroscopy spectra of (d) $\mathrm{P} L$ edge and (e) $\mathrm{O} K$ edge collected from the BP flake in (a). (f) Redox potentials of selected dopants and BP/BP oxide, relative to a standard hydrogen electrode (SHE)

\section{Results and Discussion}

BP flakes were mechanically-exfoliated from bulk crystals onto $300 \mathrm{~nm}$ thick thermal $\mathrm{SiO}_{2}$ on $\mathrm{Si}$ substrates (see experimental section for details). As the substrate is known to play an important role in the emission energy for BP, ${ }^{[37]}$ all measurements were carried out on BP flakes on identical substrates. Figure 1a shows the scanning transmission electron microscope (STEM) micrograph of a representative BP flake on a lacy carbon grid. Energy dispersive X-ray spectroscopy (EDX) was carried out on BP flakes to simultaneously obtain the planar elemental maps of phosphorus and oxygen as shown in Figure $1 \mathrm{~b}$ and 1c, respectively.
Weak EDX signals of oxygen indicate commonlyencountered surface oxidation of the BP flake after exfoliation under ambient conditions. The electron energy loss spectroscopy (EELS) spectra are collected to qualitatively assess the chemical composition of the representative BP flake. Figure 1d and e show background corrected $\mathrm{P} L$ edge and $\mathrm{O} K$ edge EELS spectra. The onset of $\mathrm{P} L_{2,3}$ edge at $132 \mathrm{eV}$ and $\mathrm{O} K$ edge at $532 \mathrm{eV}$ further confirms the surface oxidation of the BP flake. As discussed later in the manuscript, this partial surface oxidation plays a significant role in the emergence of highly intense PL in chemically doped BP.

For the chemical doping of few-layer BP, we chose four different organic charge transfer molecules, namely 

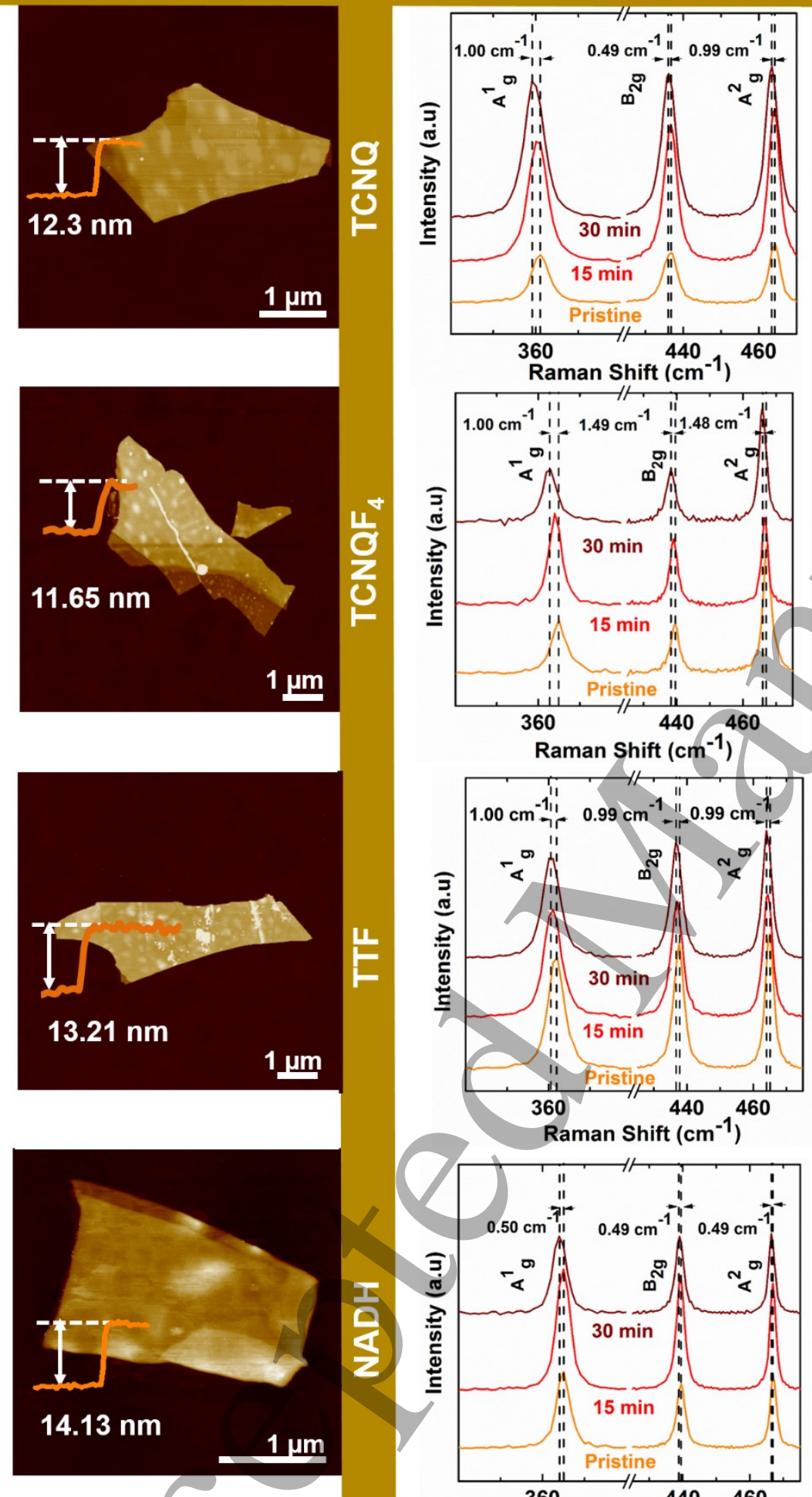

\section{PHOTOLUMINESCENCE}
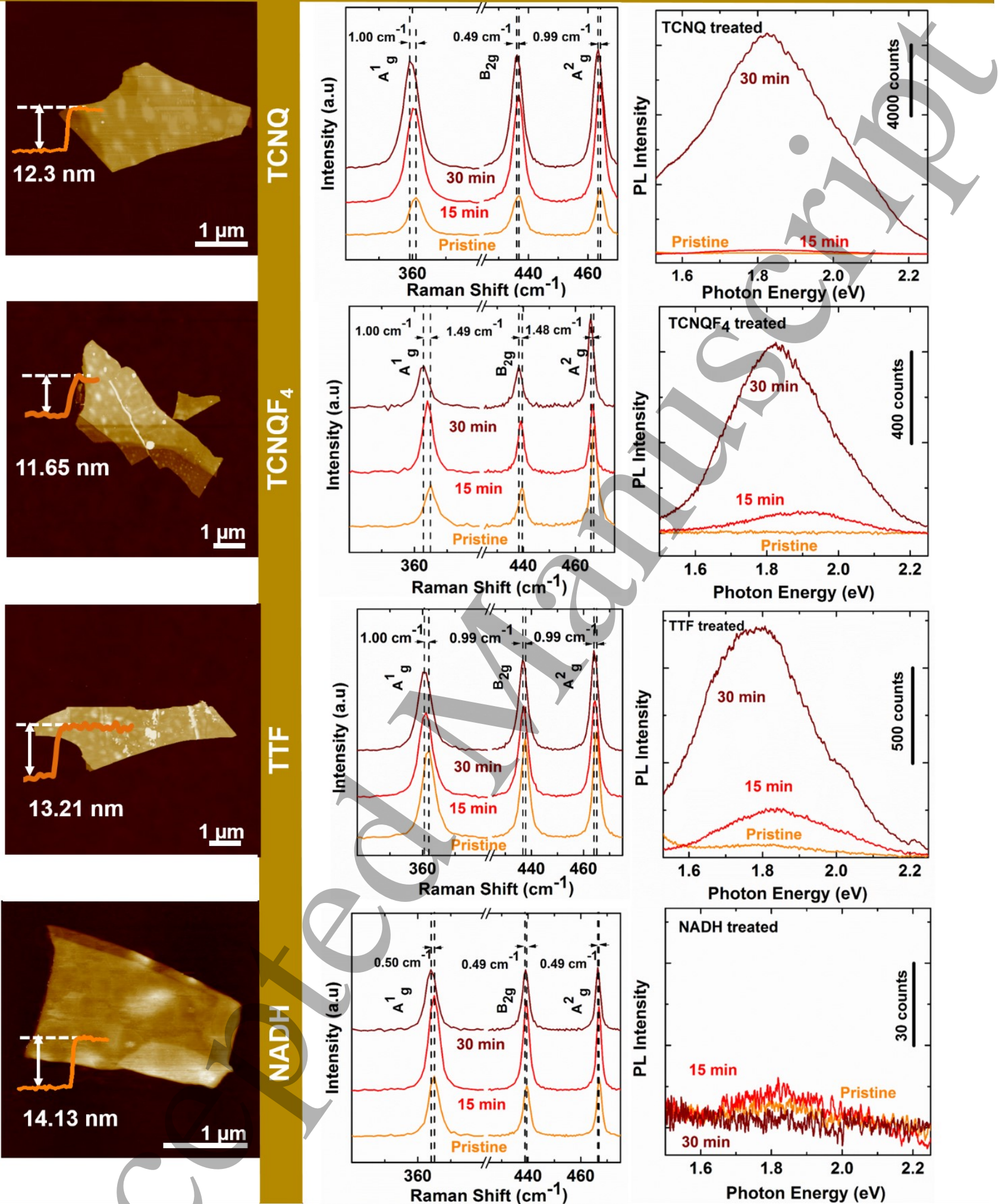

Figure 2: The AFM images (Scale bar $1 \mu \mathrm{m}$ ) of the pristine BP flakes before chemical doping, showing height of the flakes as insets. The corresponding Raman and photoluminescence peaks obtained on the BP pristine flakes, and post-dopant/BP treatment for 15 and 30 min. All measurements were under identical conditions with laser power $0.45 \mathrm{~mW}$.

\section{7,7,8,8-tetracyanoquinodimethane}

2,3,5,6-tetrafluro-7,7,8,8-tetracyanoquinodimethane

$\left(\mathrm{TCNQF}_{4}\right),{ }^{[23]}$ tetrathiafulvalene $(\mathrm{TTF}),{ }^{[38]}$ and reduced nicotinamide adenine dinucleotide (NADH) ${ }^{[39]}$ The dopant

$(\mathrm{TCNQ}),{ }^{[38]}$ molecules were chosen based on their proximity to the redox potential of few-layer $\mathrm{BP},{ }^{[7,40]}$ which makes them either $n$-type (donor) or $p$-type (acceptor) (Figure 1f) relative to BP. We chose dopant molecules such that two have redox potentials 
closer to that of BP (TCNQ and TTF) and two having redox potentials relatively further $\left(\mathrm{TCNQF}_{4}\right.$ and $\left.\mathrm{NADH}\right)$ from that of BP.

Optical images were used to identify flakes of thickness in the range of 10-15 $\mathrm{nm}$ and later confirmed by atomic force microscopy (AFM, Figure 2). ${ }^{[19]}$ BP flakes in this thickness range do not exhibit $\mathrm{PL}$ at visible wavelengths and are therefore a good starting point to ascertain the influence of chemical doping. Such freshly exfoliated BP flakes will be referred to as pristine mulitlayer BP flakes henceforth in this work. The chemical doping was performed using a drop-cast method (see experimental section for details). All optical measurements were performed after 15 and $30 \mathrm{~min}$ posttreatment. Raman spectroscopy in conjunction with photoluminescence (PL) measurements was used to assess the influence of chemical doping of BP. The measurements were carried out using a $2.33 \mathrm{eV}$ solid state green laser as the excitation energy. Typical excitation power was maintained below $0.5 \mathrm{~mW}$ to avoid any heating and nonlinear optical effects. All measurements were carried out under identical conditions with the BP flake in the same orientation. Figure 2 shows typical AFM images of the pristine BP flakes along with the evolution of the respective Raman and PL spectra for chemically doped BP. The prominent Raman peaks of BP are known to exist at $\mathrm{A}_{\mathrm{g}}^{1}\left(361 \mathrm{~cm}^{-1}\right), \mathrm{B}_{2 \mathrm{~g}}\left(437 \mathrm{~cm}^{-1}\right)$ and the $\mathrm{A}_{\mathrm{g}}^{2}$ $\left(464 \mathrm{~cm}^{-1}\right),{ }^{[41]}$ which are observed in both pre- and post-doped samples in the case of all four dopants. A closer look at the Raman spectra suggests that post-doping, a slight red-shift occurs in the BP Raman peaks for each doping step (Figure 2) which indicates an interaction of the dopant with the BP. ${ }^{[29}$ The least red shift is observed for the NADH/BP indicating minimal or no interaction with BP. Figure 2 shows the evolution of the PL spectra of pristine multilayer BP as against the doped BP systems. It is observed that while there is no PL signal from the pristine BP (10-15 nm thick) as expected. ${ }^{[20,21,42]}$ high intensity PL peaks emerge in the case of $\mathrm{TCNQF}_{4} / \mathrm{BP}, \mathrm{TCNQ} / \mathrm{BP}$ and TTF/BP within $15 \mathrm{~min}$ of treatment. As the time of treatment was increased to 30 minutes (Figure 2), the PL intensity showed a significant increase from what was observed for $15 \mathrm{~min}$ post-treatment for $\mathrm{TCNQF}_{4}, \mathrm{TCNQ}$ and TTF doped BP. Such an observation is consistent with other $2 \mathrm{D}$ material systems where the prolonged treatment time resulted in enhanced PL emission emission which would occur only if the dopant interacts with the 2D material. ${ }^{[28,43]} \mathrm{We}$ also conducted a thickness dependent analysis of the PL for BP thicknesses in the range of 7 and $20 \mathrm{~nm}$. (shown in Supplementary Figure S7). The thinner BP flakes show higher PL intensity compared to the thicker flakes which can be attributed to the enlarged bandgap of BP as the thickness reduces. This provides an opportunity to form a larger number of in-gap states post-doping. $\mathrm{NADH} / \mathrm{BP}$ did not reveal any PL enhancement even after increased doping time.
Control samples with each of the four dopants on identical substrate, but in the absence of BP flakes, are also prepared to highlight the role of the dopant/BP interaction. The PL peaks are obtained of the dopant on the bare substrate and repeated on the same spot after washing off (supplementary Figure S1). All four control samples show negligible PL response after the dopants are washed off the bare substrate. In the case of doped $\mathrm{BP}$, significant PL peaks appear close to $1.8 \mathrm{eV} \pm 0.05 \mathrm{eV}$ with distinctively different peak widths. PL in BP is known to be dominated by excitonic effects and these peaks can be identified with neutral exciton recombination. ${ }^{[37]}$ However, similar to graphene oxide, oxidation of BP is also an effective approach to control PL emission and recent theoretical studies predict that the bandgap of phosphorene oxide depends on the oxygen concentration. ${ }^{[44-46]}$ In our study, as the exfoliation is conducted in an ambient environment, an oxide layer exists on the surface as evidenced from the TEM and EELS data presented in Figure 1. While the oxide layer for BP typically does not alter the atomic layered structure of $\mathrm{BP},{ }^{[44]}$ the bandgap of the $\mathrm{BP} /$ oxide system is expected to be significantly larger than the bandgap of bulk BP. ${ }^{[44,46]}$ As shown by previous theoretical analyses, different degrees of functionalization of phosphorene by oxygen leads to various electronic structures. ${ }^{[46]}$ In our case, the dopants are adsorbed onto the surface and act as charge donors/acceptors depending on their $p$-or $n$-type nature. These dopants can therefore result in the creation of in-gap states in the BP (surface oxide) crystal which can act as recombination centres resulting in the emergence of new PL peaks. ${ }^{[47]}$ To further support this hypothesis, we acquired the PL spectra of our doped BP systems using BP layers exfoliated and preserved in an inert environment to curb the formation of any significant oxide layer (supplementary in Figure S2). It is observed (supplementary Figure S2) that no significant PL peaks emerge after treatment of BP prepared and stored in an inert environment (which therefore lacks a significant oxide layer) with the dopant. This supports the hypothesis that the formation of an oxide layer is a critical factor that governs the doping mechanism and the subsequent effect on the electronic structure of the material system.

The typical full-width half maxima (FWHM) of the PL peaks observed for the four doped BP/oxide systems lie in between 320 and $415 \mathrm{meV}$ centred at $1.8 \mathrm{eV} \pm 0.05 \mathrm{eV}$ (see supplementary Figure $\mathbf{S 3}$ for calculation). The emission spectrum of a single peak with a FWHM of $\sim 150 \mathrm{meV}$ centred at $\sim 1.3 \mathrm{eV}$ has been reported for monolayer BP. ${ }^{[19]}$ The observed PL peak from our $\sim 10 \mathrm{~nm}$ thick BP flakes doped with charge transfer molecules emerges at a relatively higher energy compared to the expected PL emission of the monolayer BP (0.6-0.8 eV ). ${ }^{[48]}$ The FWHM for the doped system (Figure S3) is larger than that reported for monolayer BP. From the uncertainty relation, a broader PL emission width indicates reduced excitonic lifetimes and hence 
increased recombination which results in a higher intensity PL emission. ${ }^{[4]}$ This broadening of the PL peak can be ascribed to the introduction of additional in gap states within the band gap of BP/oxide system resulting in the reduction of excitonic lifetimes which manifests as peak broadening in the PL spectra. ${ }^{[29]}$ These in gap states typically act as centres for carrier recombination giving rise to PL. ${ }^{[50]}$ It is noted that no PL emergence or enhancement is seen in the case of $\mathrm{NADH} / \mathrm{BP}$. NADH, has a redox potential that is significantly lower from that of $\mathrm{BP} /$ surface oxide rendering it an ineffective dopant. In addition, NADH is a large molecule relative to $\mathrm{BP}$ which may also hinder its effective adsorption to the few-layer BP surface.

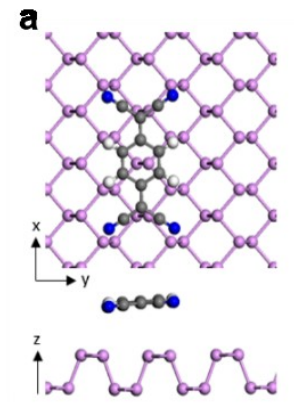

$\mathrm{BE}=-1.12 \mathrm{\theta V}$

$\Delta q=-0.34 e$
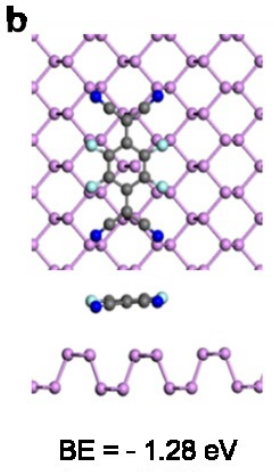

$\Delta q=-0.52 \mathrm{e}$

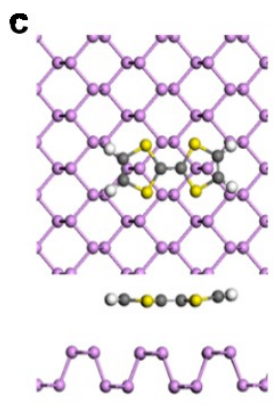

$B E=-1.03 \theta \mathrm{V}$ $\Delta q=-0.12 e$
Figure 3: The most stable structures of (a) TCNQ, (b) $\mathrm{TCNQF}_{4}$ and (c) TTF on the phosphorene basal plane as calculated using DFT. The binding energy and Bader charge transfer values were also obtained.

To obtain insights into the nature of binding of these chargetransfer dopants to BP, we performed density functional theory (DFT) calculations (Figure 3). A non-covalent interaction was observed with the strongest adsorption energy observed for $\mathrm{TCNQF}_{4}(-1.28 \mathrm{eV})$, followed by TCNQ ($1.12 \mathrm{eV})$ and TTF $(-1.03 \mathrm{eV})$, respectively. The adsorption energy being in a close range, results in smaller variations in the PL energy than expected even though the redox potential is the main parameter that governs the PL via governing the efficiency of charge transfer. It has been previously predicted that such strong interactions of charge transfer molecules with $\mathrm{BP}$ are capable of inducing surface bending of BP. ${ }^{[51]}$ It is clear that all three above mentioned $p$-type molecules exhibit a strong interaction and considerable charge transfer with BP which manifest as a change in key intrinsic properties as indicated by a drastic change in the PL spectra. NADH could not be theoretically assessed owing to the size of the molecule and prohibitively long computational cost to run such a calculation. These experimental results provide compelling evidence in regard to the chemical doping mechanism for fewlayer BP. As shown in Figure 1f, the reported chemical potential of multi-layer (thickness range 10 to $15 \mathrm{~nm}$ ) BP ranges from 0.20 to $0.36 \mathrm{eV},{ }^{[7,40,52,53]}$ which is smaller than the reduction potentials of $0.84 \mathrm{eV}$ for $\mathrm{TCNQF}_{4},{ }^{[23]} 0.46 \mathrm{eV}$ for TCNQ ${ }^{[38]}$ and $0.30 \mathrm{eV}$ for TTF relative to a standard hydrogen electrode (SHE). ${ }^{[38]}$ The chemical potential of the surface $\mathrm{BP} /$ oxide ranges from $0.56 \mathrm{eV}$ to $0.40 \mathrm{eV}^{[54]}$, which is larger than that of pristine BP. As such, these $p$-type dopants function as electron acceptors for BP. In contrast, the $n$-type dopant $(\mathrm{NADH})$ functions as an electron donor due to its lower redox potential $(-0.42 \mathrm{eV})^{[39]}$. The difference in chemical potential between BP and the $p$ - or $n$-type dopants induces an electron extraction or injection, respectively, which influences the number of excitons and their lifetimes in the dopant/oxide/ BP system. ${ }^{[51]}$

The adsorption geometry of TCNQ on BP/agrees with previous DFT calculations by Jing et al..$^{[51]}$ We observe that $\mathrm{TCNQF}_{4}$, which has not been calculated before, adsorbs in the same orientation and site as the TCNQ but with a stronger binding energy. For both structures the 6-membered ring of the molecule is located above an upper layer $\mathrm{P}$ atom. These two molecules are also stable when oriented along the $y$ direction (Supplementary Figure S5). The difference in binding energy for both molecules compared to the most stable orientation is only $0.03 \mathrm{eV}$, indicating they may be adsorbed in multiple possible orientations on the BP. Previously calculated band structures for the TCNQ and TTFadsorbed systems have shown that for the TCNQ/BP system, a new state is introduced on doping, which is very close to the valence band edge of phosphorene. ${ }^{[50,51]}$ These states can also be seen in the calculated DOS (supplementary Figure S6) which also affirm the presence of such states for TCNQF 4 . These acceptor states are found below the Fermi level of BP closer to the valence band as expected for a $p$-type dopant. ${ }^{[50,55]}$ These observations are consistent with the above conclusions that TCNQ and $\mathrm{TCNQF}_{4}$ are both effective electron extraction molecules. As a result, these shallow ingap states, introduced after doping, allow for an enhanced excitonic recombination when excited by light. However, the effect of TTF on the in-gap states is projected to be slightly different. In the TTF/BP system, a new state is introduced in the middle of the band gap region as a deep gap level, below the Fermi level, far from the conduction band edge of BP, which is not typical of for this $n$-type dopant. ${ }^{[50]}$ (Supplementary Figure S6) It is therefore introducing deep states compared to the shallow states introduced by $\mathrm{TCNQF}_{4}$ and TCNQ. Particularly, due to relatively large charge transfer between $\mathrm{TCNQF}_{4}$ or TCNQ and BP, the molecular levels of $\mathrm{TCNQF}_{4}$ and TCNQ distort more severely than those of TTF. ${ }^{[51]}$ Similar results are found in other 2D structures such as $\mathrm{BN}$ and $\mathrm{MoS}_{2}$ sheets post-modification with organic molecules. ${ }^{[28,56,57]}$ Although the redox potential graph shown in Figure 1f suggests that TTF should be a $n$-type dopant for $\mathrm{BP}$, our DFT calculations as well as previous theoretical analysis $^{[50,51]}$ show that the binding of TTF to the BP surface is highly influenced by the orientation of adsorption on BP with the most stable structure having an adsorption energy of $-1.03 \mathrm{eV}$ binding energy. The same trend of PL behaviour is 
observed when BP is treated for 15 min (which is repeated on several samples). In the most stable orientation, the two fivemembered rings of TTF are located above the two $\mathrm{P}$ atoms in the upper atomic plane. We calculated further 5 stable orientations of TTF on BP (supplementary Figure S5). For the next 2 most stable structures, TTF is oriented along the $x$ direction with the energy difference between these structures and the most stable orientation being only $0.02 \mathrm{eV}$. This indicates that TTF can adsorb in multiple orientations and sites on the BP. For the other 3 stable orientations, TTF is oriented along the $x y$-direction and are $0.04 \mathrm{eV}, 0.07 \mathrm{eV}$, and $0.10 \mathrm{eV}$ less stable. Three of these structures have not been determined previously. For all orientations, the binding energy values indicate that TTF is chemisorbed on the surface and is located approximately $3.3 \AA$ above the upper plane of $\mathrm{P}$ atoms.
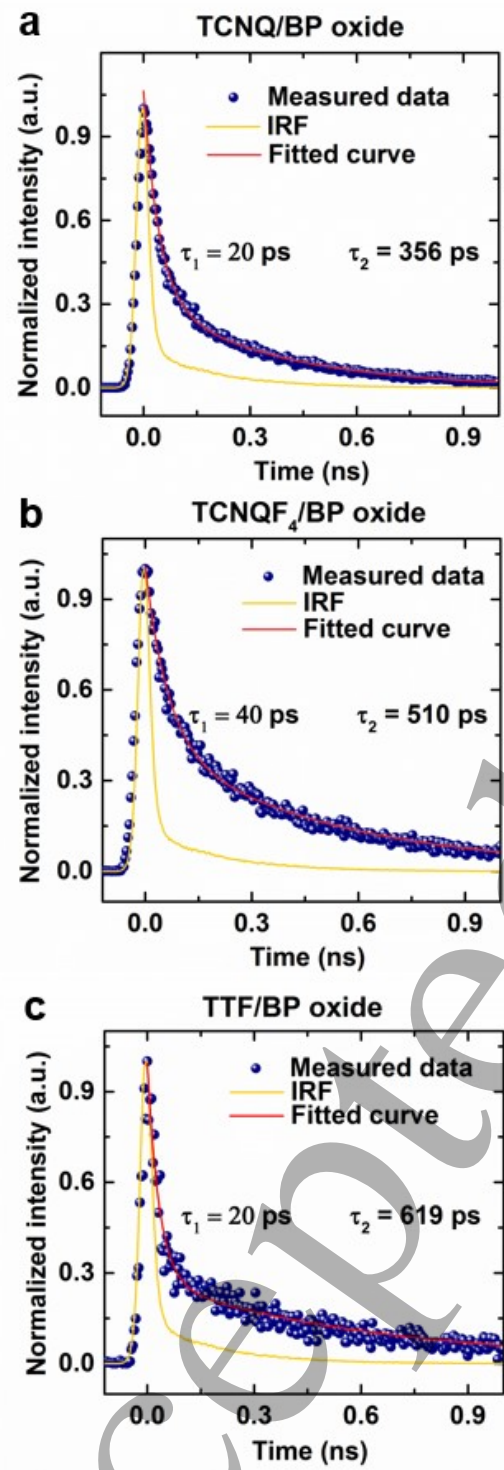

Figure 4. The time resolved photoluminescence lifetime measurements of (a) TCNQ/BP oxide, (b) TCNQF4/BP oxide and (c) TTF/BP oxide with the fitted curve and instrument response function (IRF). All data was acquired using $532 \mathrm{~nm}$ power source of $0.45 \mathrm{~mW}$ laser power on the 15 minute doped samples.

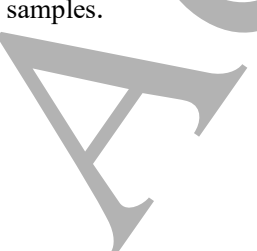

In addition, to experimentally confirm the introduction of ingap states, we performed time resolved photoluminescence lifetime measurements (shown in Figure 4) the carrier lifetime measurements indicate the average time the carrier stays in the excited state before returning to the ground state. The $\tau_{1}$ is the characteristic of the direct transition from excited state to the ground state whereas $\tau_{2}$ is the characteristic of the transition from the intermediate states, here the in-gap states to the ground state. ${ }^{[58]}$ From the measured values of $\tau_{2}$ for the doped $\mathrm{BP}$, the lifetimes of $356 \mathrm{ps}$ (TCNQ), $510 \mathrm{ps}\left(\mathrm{TCNQF}_{4}\right)$ and 619 ps (TTF) indicate that the in-gap states are introduced due to doping in the BP/oxide system band gap resulting in the emergence of strong PL. This matches with the obtained PL intensity trend for the doped $\mathrm{BP}$, as a longer transition time results in a proportionally higher intensity of the PL. This is also in line with the DFT calculations which suggested the formation of in-gap states due to doping. The three dopants have slightly different measured lifetime values (Supplementary Figure S8), which might be related to the carrier doping levels. ${ }^{[59,60]}$ The lifetime values obtained for the doped BP are higher than that reported for the monolayer BP at 220 ps. $^{[22]}$
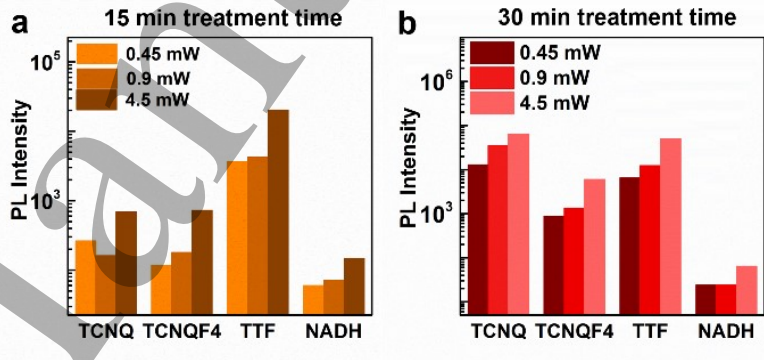

Figure 5: The logarithmic graphs show the power-dependent PL with the dopants on BP for both the two doping steps performed. PL Intensities at laser powers $0.45 \mathrm{~mW}, 0.90 \mathrm{~mW}$, and $4.50 \mathrm{~mW}$ for (a) 15 min treatment time with the inset showing magnified PL intensities of the dopants TCNQ, TCNQF and NADH. (b) The PL intensities of the dopants/BP for 30 min treatment time. The inset shows the zoomed in PL intensities for NADH at the different laser powers. The graph indicates that the intensities increase with the increased laser power.

In Figure 5, we present the dependence of the PL intensity as a function of excitation power. The effect of excitation power was studied by acquiring the PL spectra by sequentially varying the laser power from $0.45 \mathrm{~mW}$ to $4.50 \mathrm{~mW}$. As the power is increased, the magnitude of the PL enhancement also increases (Figure 4 and supplementary Figure S4) in agreement with the power law. ${ }^{[22,37,61]}$ This further confirms that the PL emerging from the doped BP is due to the chemical interactions and not just laser- induced. It is also noted that for laser powers beyond $4.5 \mathrm{~mW}$, the BP flakes suffered from laser-induced degradation with visible black spots making them unusable for any further measurements.

\section{Conclusions}


In conclusion, we have demonstrated that chemical doping of BP/oxide with carefully chosen $p$-type molecules is an effective pathway to engineer its optical properties at room temperature. The formation of a surface oxide layer on BP results in changes to the electronic structure, wherein the dopants can introduce acceptor and donor states resulting in the emergence of very strong PL emissions. This, to our knowledge, is the first observation of such highly intense PL from multilayered BP in the visible spectrum. Our experimental results are supported with theoretical calculations which indicate the formation of additional gap states facilitating the change in intrinsic characteristics upon doping. This simple yet powerful doping methodology opens a pathway for the deployment of multilayer BP in a range of optoelectronic applications.

\section{Experimental Section}

4.1 Sample preparation and storage: The BP flakes are mechanically exfoliated from bulk BP crystals (Smart Elements) using blue PVC tape (Nitto) onto a plasma-cleaned $\mathrm{Si}$ substrate with dry thermal $\mathrm{SiO}_{2}(300 \mathrm{~nm})$. To prevent the few-layer BP from photo-oxidation, the samples are prepared and characterised in an UV-deficient environment, as UV is responsible for photo-oxidation induced degradation of BP. ${ }^{[25,62-64]}$

4.2 Dopants preparation and doping: Four different organic molecules are employed as dopants to assess their influence on the PL emission characteristics of few-layer BP. The dopants are chosen based on their proximity to the redox potential of few-layer BP which makes them $n$-type or $p$-type dopants (Figure 1) relative to BP. 7,7,8,8-tetracyanoquinodimethane (TCNQ ${ }^{[38]}, 2,3,5,6$-tetrafl uro-7,7,8,8-Tetracyanoquinodimethane $\left(\mathrm{TCNQF}_{4}\right)^{[23]}$, and tetrathiafulvalene (TTF) ${ }^{[38]}$ are chosen as the $p$-type dopants and reduced nicotinamide adenine dinucleotide (NADH) ${ }^{[39]}$ as an $n$-type dopant. The solution-based chemical doping is performed using a drop-cast method. The dopant solution is pipetted onto the selected BP flakes, given defined interaction durations, before being washed in acetonitrile. The concentration of each dopant was kept constant at $0.1 \mathrm{mM}$.

4.3 Theoretical calculations: The calculations were performed using density functional theory (DFT) as implemented in the Vienna ab initio simulation package ${ }^{[65-67]}$, using the generalised-gradient approximation and Perdew, Burke and Ernzerhof (PBE) exchange-correlation functional, and the projector-augmented wave method. ${ }^{[68,69]}$ To account for van der Waals forces, the Grimme D3 ${ }^{[70]}$ method was used. A plane-wave basis with an energy cut-off of $420 \mathrm{eV}$ was employed with a Monkhorst-Pack k-point mesh of $4 \times 4 \times 1$. The basal plane of phosphorene was modelled using a $[6 \times 4]$ super cell as described in the reports of Jing et al. and Walia et $a l .{ }^{[51,71]}$ The cell has dimensions of $19.79 \AA \times 18.49 \AA$, with a vacuum spacer of $17.00 \AA$ in the z-direction to prevent interactions between periodic cells in this direction. The TCNQ, TCNQF 4 and TTF dopant molecules were each initially adsorbed $\sim 3 \AA$ above the phosphorene surface in different initial orientations and adsorption sites. A geometry optimisation was performed for each structure keeping the lattice parameters fixed while allowing the atomic positions to relax until the total energy was converged to $10^{-4} \mathrm{eV}$ and the Hellman-Feynman force on each relaxed atom was less than $0.03 \mathrm{eV} / \AA$. The binding energy $(\mathrm{BE})$ values ${ }^{[57]}$ were calculated using the formula: $\mathrm{BE}=E_{\mathrm{M} / \mathrm{P}}-\left(E_{\mathrm{M}}-E_{\mathrm{P}}\right)$, where $E_{\mathrm{M} / \mathrm{P}}$ is the total energy of the dopant molecule (TCNQ, $\mathrm{TCNQF}_{4}$ or TTF) adsorbed on the phosphorene, $E_{\mathrm{M}}$ is the total energy of the isolated molecule and $E_{\mathrm{P}}$ is the total energy of the clean phosphorene. According to this convention, stable structures have a negative binding energy. A vibrational frequency calculation was sued to confirm that each structure was a minimum.

4.4 Time resolved lifetime measurements: This measurement was undertaken using a $532 \mathrm{~nm}$ laser pulse source (maintaining the laser power at $0.45 \mathrm{~mW}$ ) in a time domain method evaluating the decay curve after excitation with the light source. The decay trace curves were deconvoluted with respect to the instrument response and then were fitted with the equation: $\mathrm{I}=\mathrm{A} \mathrm{e}^{\left(-\frac{\mathrm{t}}{\tau_{1}}\right)}+\mathrm{B} \mathrm{e}^{\left(-\frac{\mathrm{t}}{\tau_{2}}\right)}+\mathrm{C}$, where $\mathrm{I}$ is the PL intensity, $\mathrm{A}, \mathrm{B}$, and $\mathrm{C}$ are constants, $\mathrm{t}$ is time, and $\tau_{1}$ is faster decay rate and $\tau_{2}$ is the slower decay rate favouring the population accumulation ${ }^{[72]}$, indicating emission lifetimes for different decay processes. ${ }^{[73,74]} \tau_{1}$ and $\tau_{2}$ are dominated by nonradiative and radiative channels, respectively.

\section{Acknowledgements}

The authors acknowledge support from the Australian Government Research Training Program Scholarship and the Australian Research Council for personnel and project support via DE150100909 (S.B.), FT140101285 (V.B.), DP170103477 (V.B.), DE160100023 (M.B.), and equipment funding through LE0882246, LE0989615, and LE150100001.

\section{References}

[1] Gusmao R., Sofer Z., and Pumera M. 2017 Angew. Chem. 1298164.

[2] Liu H., Du Y., Deng Y., and Peide D. Y. 2015 Chem. Soc. Rev. 442732.

[3] Eswaraiah V., Zeng Q., Long Y., and Liu Z. 2016 Small 123480.

[4] Xia F., Wang H., and Jia Y. 2014 Nat Comm 54458.

[5] Cai Y., Zhang G., and Zhang Y.-W. 2014 Sci. Rep. 4 6677. 
[6] Dhanabalan S. C., Ponraj J. S., Guo Z., Li S., Bao Q., and Zhang H. 2017 Adv Sci 41600305.

[7] Qiao J., Kong X., Hu Z.-X., Yang F., and Ji W. 2014 Nat Comm 54475.

[8] Wang C., Niu D., Liu B., Wang S., Wei X., Liu Y., Xie H., and Gao Y. 2017 J. Phys. Chem. C 121 18084.

[9] Carvalho A., Wang M., Zhu X., Rodin A. S., Su H., and Neto A. H. C. 2016 Nat Rev Mater 116061.

[10] Cui M., Guo Y., Zhu Y., Liu H., Wen W., Wu J., Cheng L., Zeng Q., and Xie L. 2018 J. Phys. Chem. C 1227551.

[11] Buscema M., Groenendijk D. J., Blanter S. I., Steele G. A., Van Der Zant H. S., and Castellanos-Gomez A. 2014 Nano Lett. 143347.

[12] Guo Z., Zhang H., Lu S., Wang Z., Tang S., Shao J., Sun Z., Xie H., Wang H., and Yu X. F. $2015 A d v$ Funct Mater 256996.

[13] Kou L., Frauenheim T., and Chen C. 2014 J. Phys. Chem. Lett. 52675.

[14] Late D. J. 2016 Micropor Mesopor Mat 225494.

[15] Hanlon D., Backes C., Doherty E., Cucinotta C. S., Berner N. C., Boland C., Lee K., Harvey A., Lynch P., and Gholamvand Z. 2015 Nat Comm 68563.

[16] Li L., Yu Y., Ye G. J., Ge Q., Ou X., Wu H., Feng D., Chen X. H., and Zhang Y. 2014 Nat. Nanotechnol 9372.

[17] Buscema M., Groenendijk D. J., Blanter S. I., Steele G. A., Van Der Zant H. S., and Castellanos-Gomez A. 2014 Nano Lett. 143347.

[18] Buscema M., Groenendijk D. J., Steele G. A., Van Der Zant H. S., and Castellanos-Gomez A. 2014 Nat Comm 54651.

[19] Wang X., Jones A. M., Seyler K. L., Tran V., Jia Y., [40] Zhao H., Wang H., Yang L., Xu X., and Xia F. 2015 Nat. Nanotechnol 10517.

[20] Castellanos-Gomez A., Vicarelli L., Prada E., Island J. O., Narasimha-Acharya K., Blanter S. I., Groenendijk D. J., Buscema M., Steele G. A., and Alvarez J. 2014 2D Mater 1025001.

[21] Zhang S., Yang J., Xu R., Wang F., Li W., Ghufran M., Zhang Y.-W., Yu Z., Zhang G., and Qin Q. 2014 ACS Nano 89590.

[22] Yang J., Xu R., Pei J., Myint Y. W., Wang F., Wang Z., Zhang S., Yu Z., and Lu Y. 2015 Light-Sci Appl 4312.

[23] O'Connell M. J., Eibergen E. E., and Doorn S. K. 2005 Nat Mater 4412.

[24] Kuriakose S., Ahmed T., Balendhran S., Collis G. E., Bansal V., Aharonovich I., Sriram S., Bhaskaran M., and Walia S. 2018 Appl Mater Today. 12244.

[25] Kuriakose S., Ahmed T., Balendhran S., Bansal V., Sriram S., Bhaskaran M., and Walia S. 2018 2D Mater 5032001.

[26] Wang G., Slough W. J., Pandey R., and Karna S. P. 2016 2D Mater 3025011.

[27] Elbadawi C., Tormo Queralt R., Xu Z.-Q., Bishop J., Ahmed T., Kuriakose S., Walia S., Toth M.,
Aharonovich I., and Lobo C. J. 2018 ACS Appl Mater Inter 1024327.

[28] Mouri S., Miyauchi Y., and Matsuda K. 2013 Nano Lett. 135944.

[29] Vishnoi P., Rajesh S., Manjunatha S., Bandyopadhyay A., Barua M., Pati S. K., and Rao C. 2017 ChemPhysChem 182985.

[30] Farmer D. B., Golizadeh-Mojarad R., Perebeinos V., Lin Y.-M., Tulevski G. S., Tsang J. C., and Avouris P. 2008 Nano Lett. 9388.

[31] Du Y., Yang L., Zhou H., and Peide D. Y. 2016 IEEE Electron Device Lett. 37429.

[32] Late D. J., Ghosh A., Chakraborty B., Sood A., Waghmare U. V., and Rao C. 2011 J. Exp. Nanosci. 6641.

[33] Ramanathan R., Pearson A., Walia S., Kandjani A. E., Mohammadtaheri M., Bhaskaran M., Sriram S., Bhargava S. K., and Bansal V. 2018 Appl Mater Today. 1012.

[34] Mohammadtaheri M., Ramanathan R., and Bansal V. 2016 Catalysis Today 278319.

[35] Siu M. C., Anderson S. R., Mohammadtaheri M., Ahmed T., Walia S., Ramanathan R., and Bansal V. 2017 Adv Mater Interfaces 41700097.

[36] Guo Z., Chen S., Wang Z., Yang Z., Liu F., Xu Y., Wang J., Yi Y., Zhang H., and Liao L. $2017 A d v$ Mater 29.

[37] Surrente A., Mitioglu A., Galkowski K., Tabis W., Maude D., and Plochocka P. 2016 Phys. Rev. B 93 121405.

Torrance J. B. 1979 Acc. Chem. Res. 1279.

Kim K. K., Kim S. M., and Lee Y. H. 2016 Acc. Chem. Res. 49390.

Low T., Rodin A., Carvalho A., Jiang Y., Wang H., Xia F., and Neto A. C. 2014 Phys. Rev. B 90075434. Feng Y., Zhou J., Du Y., Miao F., Duan C.-G., Wang B., and Wan X. 2015 J. Phys. Condens. Matter 27 185302.

[42] Ryder C. R., Wood J. D., Wells S. A., and Hersam M. C. 2016 ACS Nano 103900.

[43] Cong C., Shang J., Wang Y., and Yu T. 2018 Adv Opt Mater 61700767.

[44] Gan Z., Sun L., Wu X., Meng M., Shen J., and Chu P. K. 2015 Appl. Phys. Lett. 107021901.

[45] Ziletti A., Carvalho A., Campbell D. K., Coker D. F., and Neto A. C. 2015 Phys Rev Lett 114046801.

[46] Ziletti A., Carvalho A., Trevisanutto P., Campbell D., Coker D., and Neto A. C. 2015 Phys. Rev. B 91 085407.

[47] Cai B., Zhang S., Yan Z., and Zeng H. 2015 ChemNanoMat 1542.

[48] Liang L., Wang J., Lin W., Sumpter B. G., Meunier V., and Pan M. 2014 Nano Lett. 146400.

[49] Hartland G. V. 2011 Chem. Rev. 1113858.

[50] Zhang R., Li B., and Yang J. 2015 J. Phys. Chem. C 1192871.

[51] Jing Y., Tang Q., He P., Zhou Z., and Shen P. 2015 Nanotechnology 26095201. 
[52] Sofer Z., Sedmidubský D., Huber S̆., Luxa J., Bouša D., Boothroyd C., and Pumera M. 2016 Angew. Chem. Int. Ed. 553382.

[53] Ran J., Zhu B., and Qiao S. Z. 2017 Angew. Chem. Int. Ed. 5610373.

[54] Zhou Q., Chen Q., Tong Y., and Wang J. 2016 Angew. Chem. 12811609.

[55] Cui S., Pu H., Wells S. A., Wen Z., Mao S., Chang J., Hersam M. C., and Chen J. 2015 Nat Comm 6 8632.

[56] Tang Q., Zhou Z., and Chen Z. 2011 J. Phys. Chem. C 11518531 .

[57] Shakourian-Fard M., Kamath G., and Jamshidi Z. 2014 J. Phys. Chem. C 11826003.

[58] Cheng C., Li J., and Cheng X. 2017 Journal of Luminescence 188252.

[59] Wang H., Zhang C., Chan W., Manolatou C., Tiwari S., and Rana F. 2016 Phys. Rev. B 93.

[60] Meng X., Wang X., Cheng Z., Tian N., Lang M. C., Yan W., Liu D., Zhang Y., and Wang P. 2018 ACS Appl Mater Inter 1031136.

[61] Tran V., Soklaski R., Liang Y., and Yang L. 2014 Phys. Rev. B 89235319.

[62] Ahmed T., Balendhran S., Karim M. N., Mayes E. L., Field M. R., Ramanathan R., Singh M., Bansal V., Sriram S., and Bhaskaran M. 2017 npj 2D Mater App 118.

[63] Favron A., Gaufrès E., Fossard F., PhaneufL'Heureux A.-L., Tang N. Y., Lévesque P. L., Loiseau A., Leonelli R., Francoeur S., and Martel R. 2015 Nat Mater 14826.

[64] Jeong M.-H., Kwak D., Ra H.-S., Lee A.-Y., and Lee J.-S. 2018 ACS Appl Mater Inter 1019069.

[65] Kresse G. and Furthmüller J. 1996 Comput. Mater. Sci 615.

[66] Kresse G. and Furthmüller J. 1996 Phys. Rev. B 54 11169.

[67] Kresse G. and Hafner J. 1993 Phys. Rev. B 4813115.

[68] Blöchl P. E. 1994 Phys. Rev. B 5017953.

[69] Perdew J. P., Burke K., and Ernzerhof M. 1996 Phys Rev Lett 773865.

[70] Grimme S., Antony J., Ehrlich S., and Krieg H. 2010 J Chem Phys 132154104.

[71] Walia S., Balendhran S., Ahmed T., Singh M., ElBadawi C., Brennan M. D., Weerathunge P., Karim M., Rahman F., Rassell A., Duckworth J., Ramanathan R., E Collis G., J Lobo C., Toth M., Christopher Kotsakidis J., Weber B., Fuhrer M., M Dominguez-Vera J., JS Spencer M., Aharonovich I., Sriram S., Bhaskaran M., and Bansal V. 2017 Adv Mater 291700152.

[72] Wei T., Tian Y., Chen F., Cai M., Zhang J., Jing X., Wang F., Zhang Q., and Xu S. 2014 Sci. Rep. 46060.

[73] Liu X., Yu H., Ji Q., Gao Z., Ge S., Qiu J., Liu Z., Zhang Y., and Sun D. 2016 2D Mater 3014001.

[74] Pei J., Yang J., Wang X., Wang F., Mokkapati S., Lü T., Zheng J.-C., Qin Q., Neshev D., and Tan H. H. 2017 ACS Nano 117468. 


\section{Supporting Information}

\section{Generating strong room-temperature photoluminescence in black phosphorus using organic molecules}

Sruthi Kuriakose, Taimur Ahmed, Patrick D. Taylor, Yi Zhu, Michelle J.S. Spencer, Sivacarendran Balendhran, Yuerui Lu, Vipul Bansal,* Sharath Sriram, Madhu Bhaskaran and Sumeet Walia*

S1: The PL comparison against the substrate and the dopants on the substrate.

a
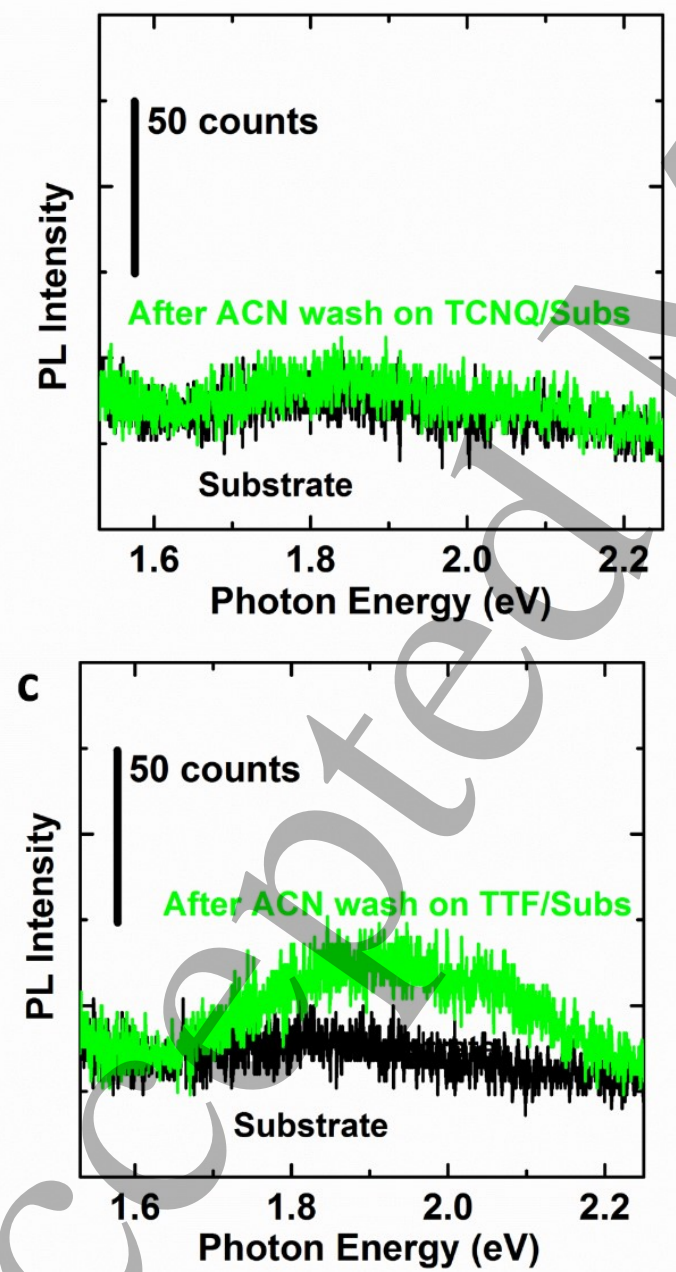

b

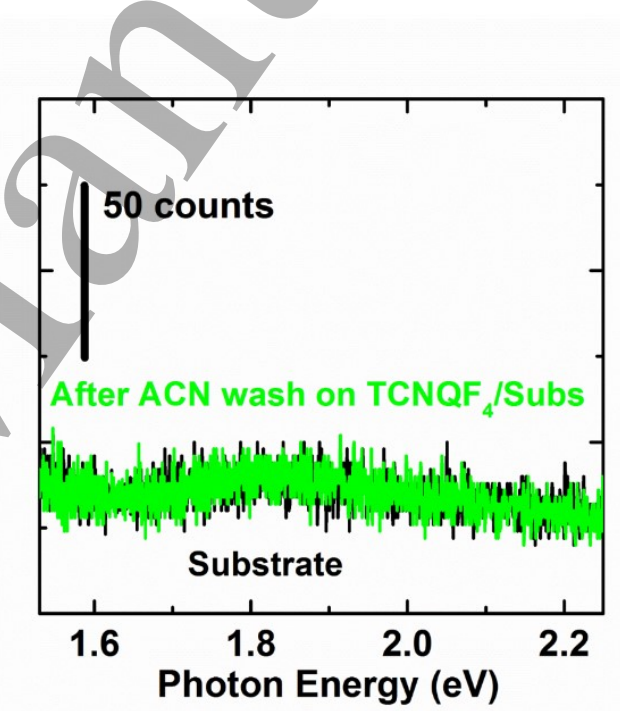

d

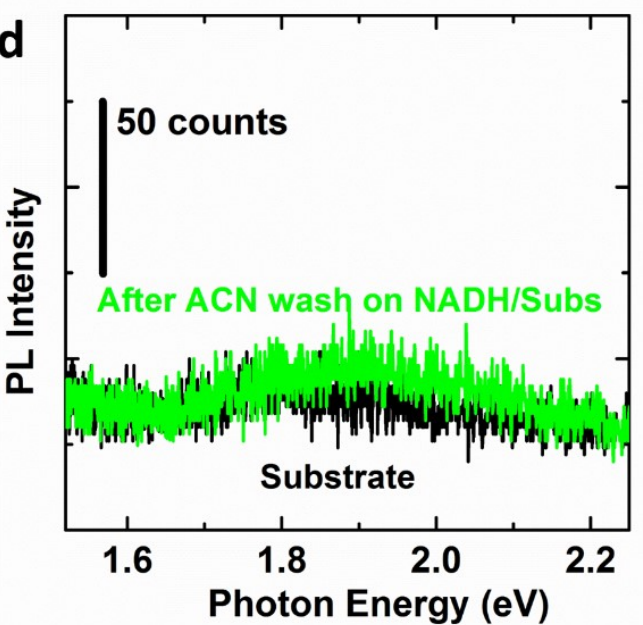

Figure S1: The graphs (raw data) shows comparison of $\mathrm{PL}$ of the bare $\mathrm{SiO}_{2}$ substrate (in black) and the substrates washed (in green) with acetonitrile $(\mathrm{ACN})$ after being treated with the dopants. (a) $\mathrm{TCNQ} / \mathrm{SiO}_{2}$, (b) $\mathrm{TCNQF}_{4} / \mathrm{SiO}_{2}$ (c) $\mathrm{TTF} / \mathrm{SiO}_{2}$ and (d) NADH/SiO 2 . The vertical scale bar is 50 counts of PL intensity. It is evident from this PL comparison that the PL of the dopants/BP flake emerge from the interaction of the BP flake and the dopant. 


\section{S2: PL of the pristine and treated samples measured under inert environment to prevent the} oxide layer formation on BP.

a

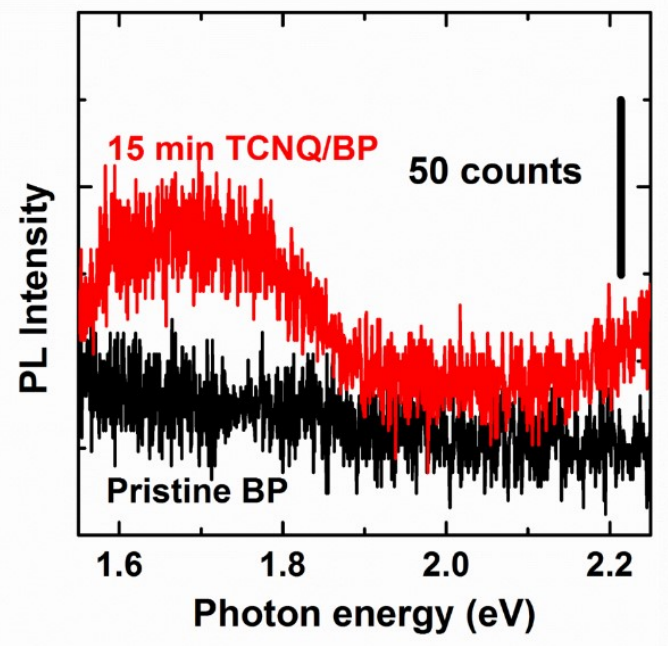

b

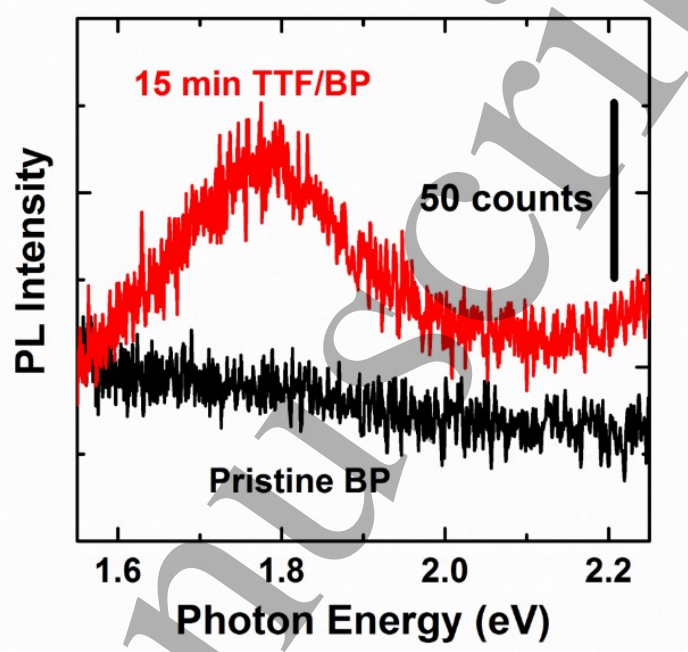

Figure S2: The graph shows the PL of the pristine BP and the treated BP with TCNQ (a) and TTF (b) in measured in inert conditions, showing a negligible emergence of PL from the dopants/BP interaction as observed in the ambient conditions.

BP was exfoliated in photolithography room (yellow room) to prevent any interaction with UV light. The exfoliated samples were immediately placed in a closed chamber with argon gas flow to avoid any interaction with the ambient air. The PL observed in these samples were negligible in comparison to the samples prepared in ambient conditions. As such, these results confirm that the significantly enhanced PL emerged from the treated BP in ambient atmosphere, is due to the presence of the surface oxide layer on BP, which has a larger band gap than that of pristine BP. The interaction of the dopants with BP (with surface oxide formed in ambience) gives a strong PL by introducing donor/acceptor states within the band gap of BP. 


\section{S3: Full width at half maximum (FWHM) for the doped BP:}
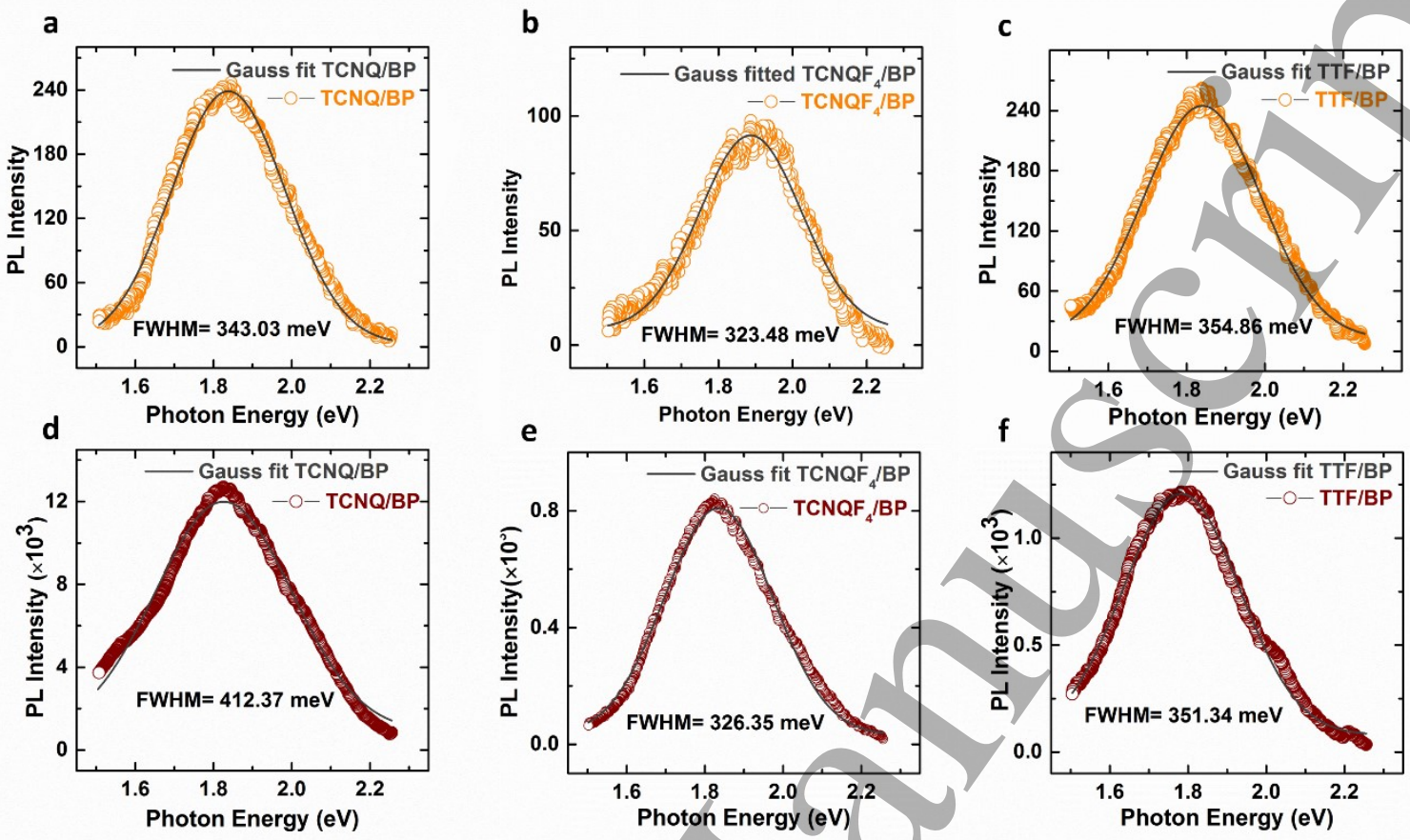

Figure S3: The graphs show the PL of the doped BP in ambience and their gauss fit curves. The graphs (a-c) show PL of the doped BP for 15 minutes and their FWHM calculated from their gauss fit curve. The graphs (d-f) shows PL of the doped BP in ambience for 30 minutes with their FWHM. The highest PL peak intensity of the doped BP show a broader PL width indicating the introduction of the dopant states during the interaction. The FWHM was calculated using the formula: $y=y_{0}+\frac{A}{w \sqrt{\pi / 2}} e^{-2 \frac{\left(x-x_{c}\right)^{2}}{w^{2}}}$ for the doped BP after fitting the PL curve. 
S4: PL of the pristine and treated samples measured at $0.9 \mathrm{~mW}$.

a

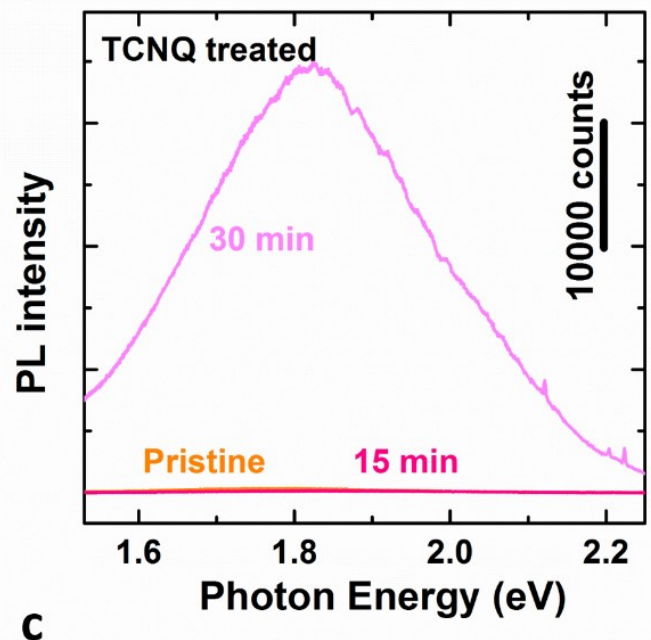

C

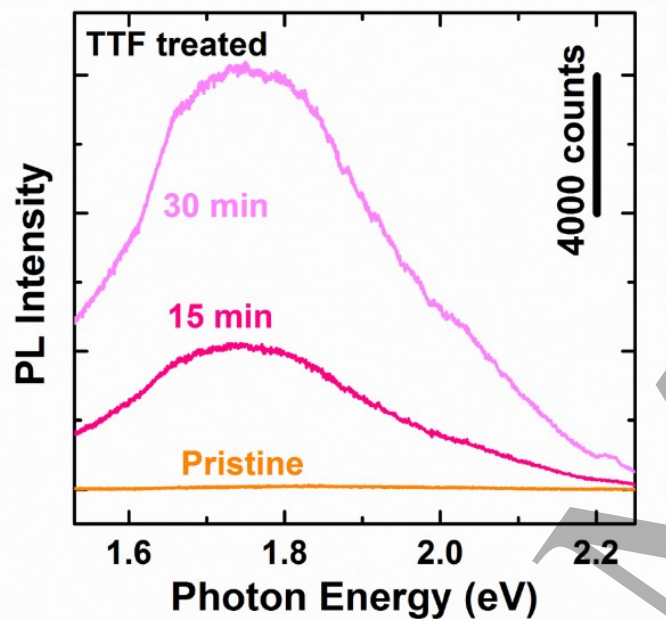

b

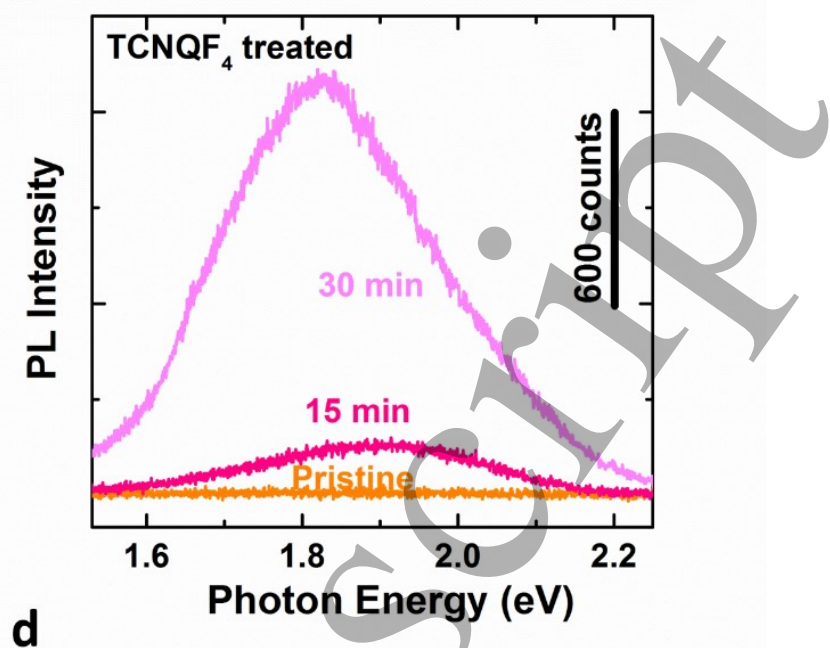

d

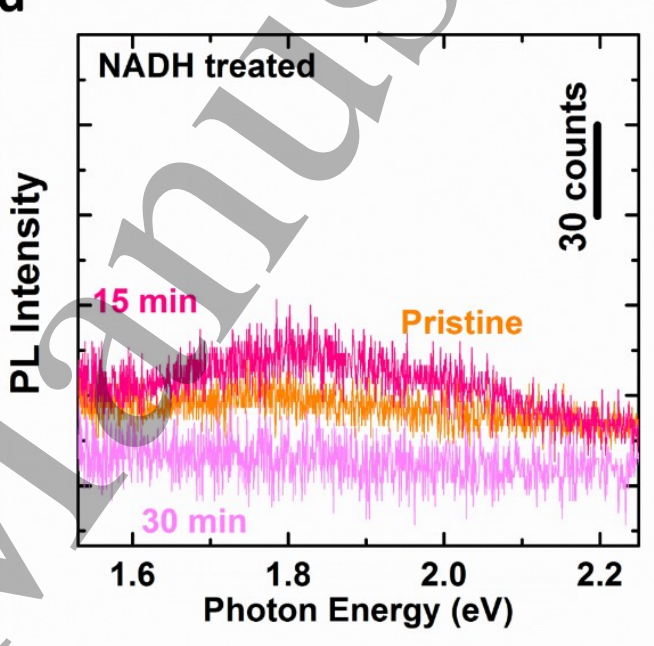

Figure S4: The graphs (raw data) show the comparison of the PL of the pristine and doped BP flakes (a) TCNQ/BP (b) $\mathrm{TCNQF}_{4} / \mathrm{BP}$ (c) TTF/BP and (d) NADH/BP measured at $0.90 \mathrm{~mW}$ power of the laser for both 15 and 30 min doping steps. 
S5: Optimised structures of the organic charge transfer molecules on the phosphorene monolayer.

a
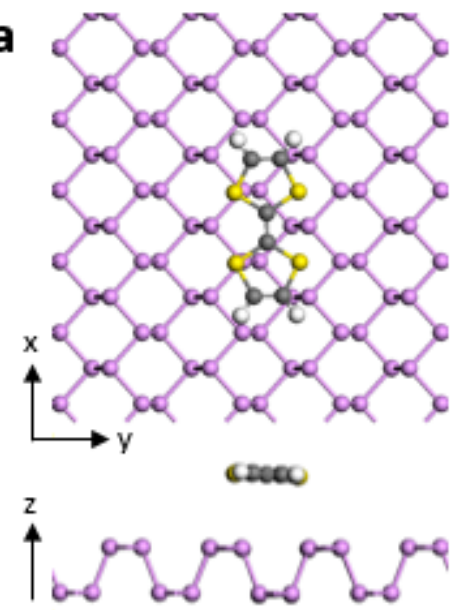

$\mathrm{BE}=-1.01 \mathrm{eV}$ b

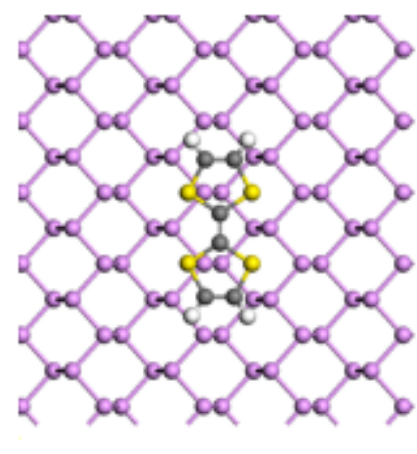

ceceo
C

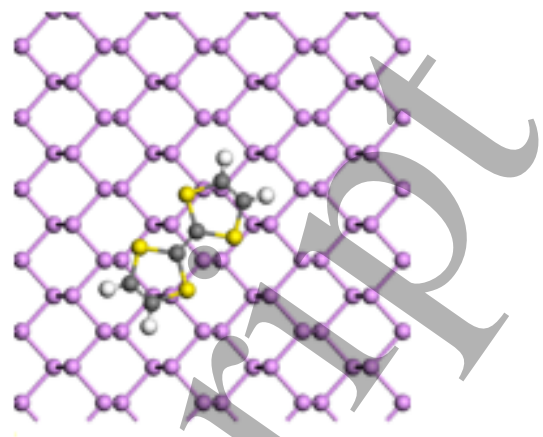

d

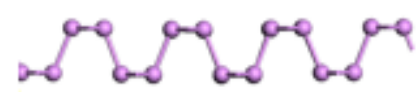

$\mathrm{BE}=-1.01 \mathrm{eV}$

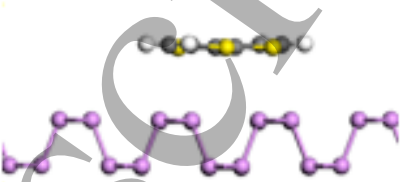

$B E=-0.99 \mathrm{eV}$

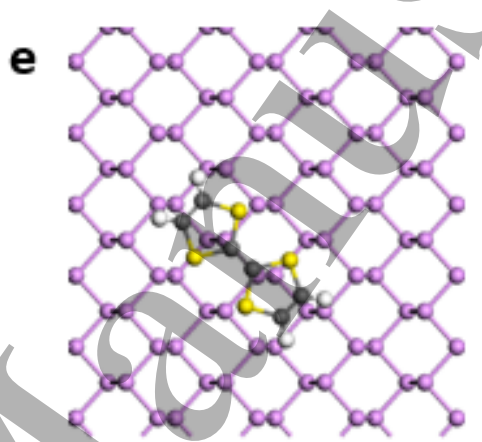

ocorescoc

cos-es-coc
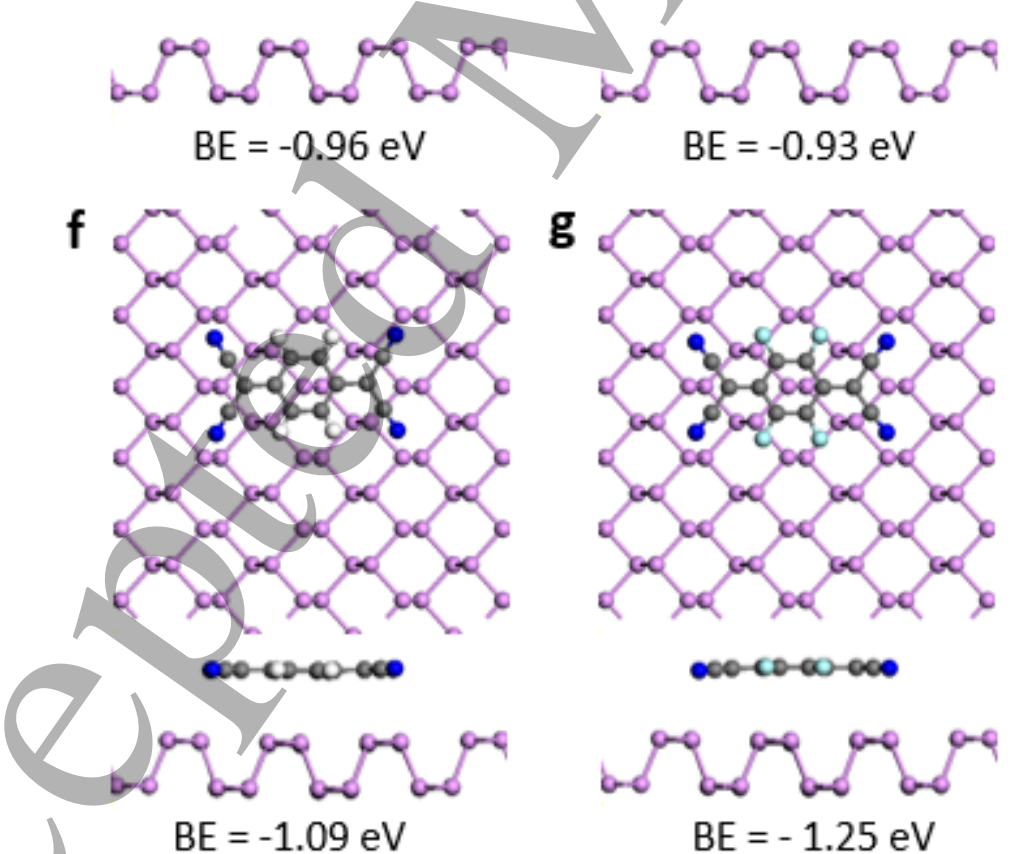

Figure S5: Top and side views of less stable optimised geometries of TTF/phosphorene (a-e), TCNQ/phosphorene (f) and $\mathrm{TCNQF}_{4} /$ phosphorene $(\mathrm{g})$. BE = Binding Energy. 
S6: Calculated Density of States for doped BP.

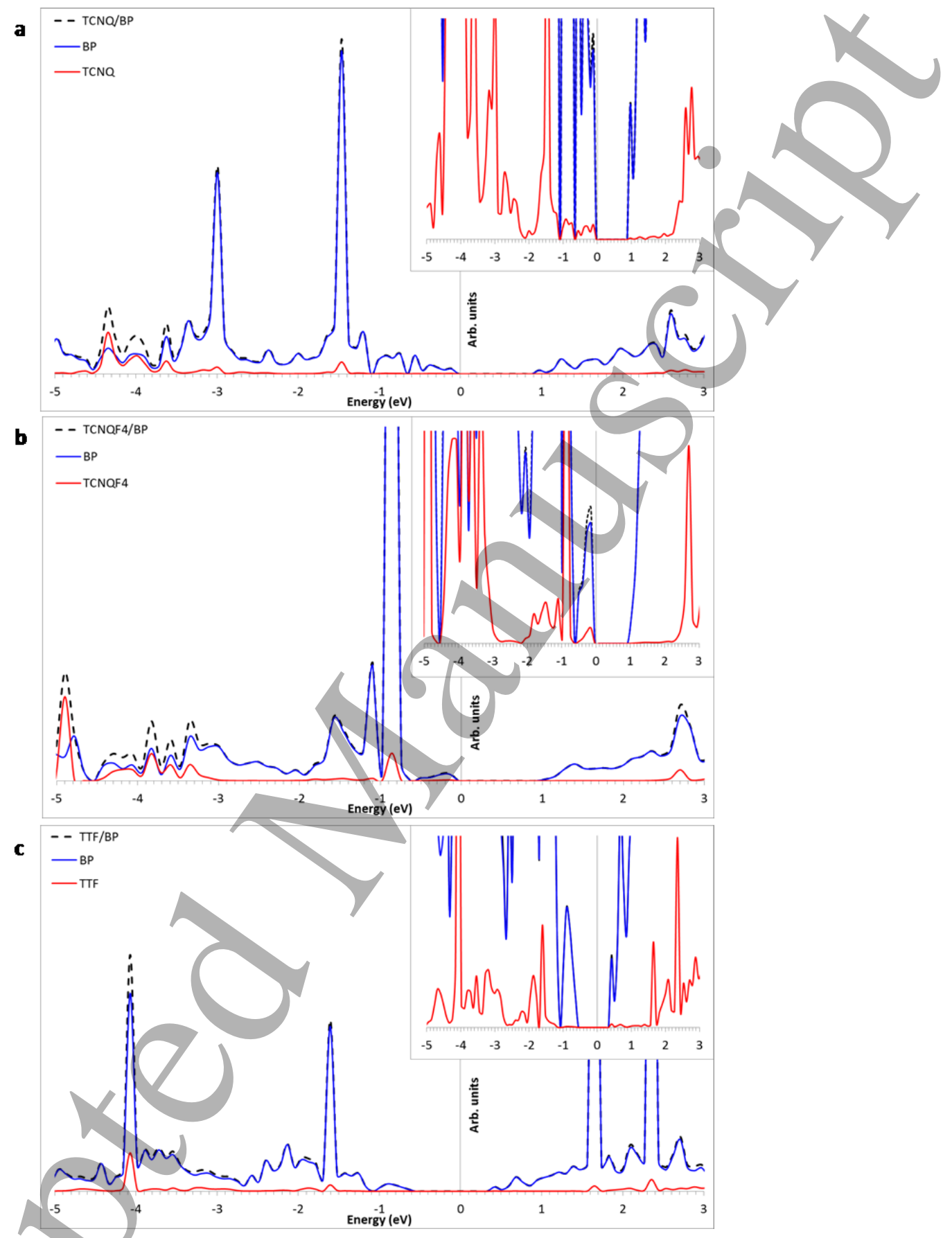

Figure S6: Partial density of states (PDOS) for TCNQ (a), $\mathrm{TCNQF}_{4}$ (b) and TTF (c) adsorbed on BP resolved to the BP atom and the donor molecule states. 


\section{S7: Thickness dependent PL emission.}

a

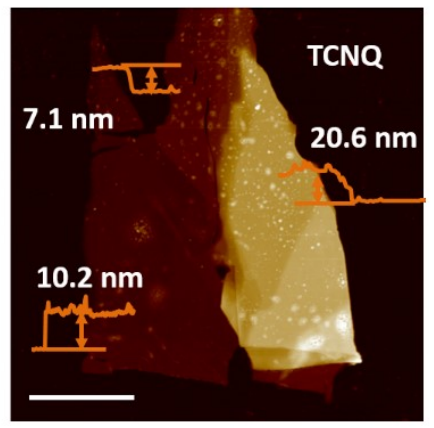

d

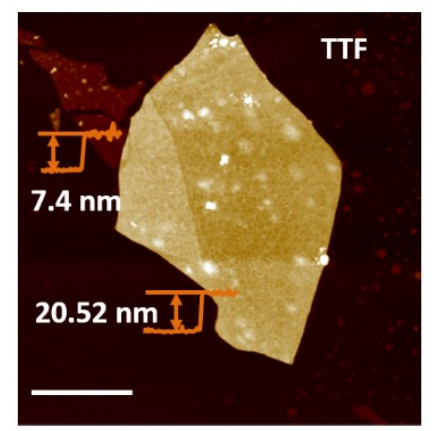

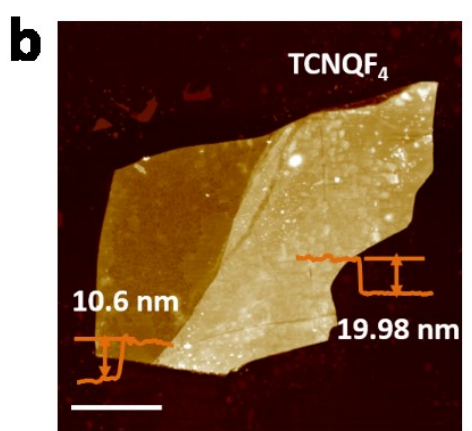

e

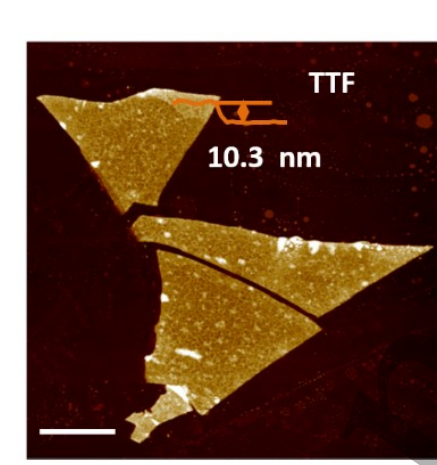

$\mathbf{C}$

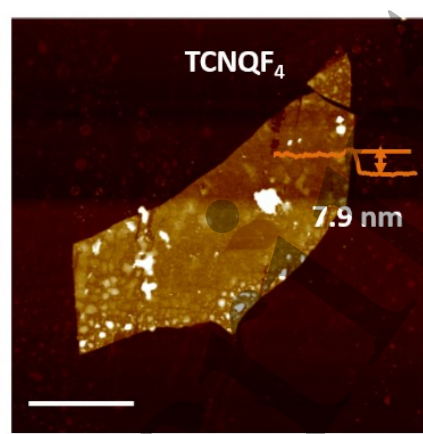

$\mathbf{f}$

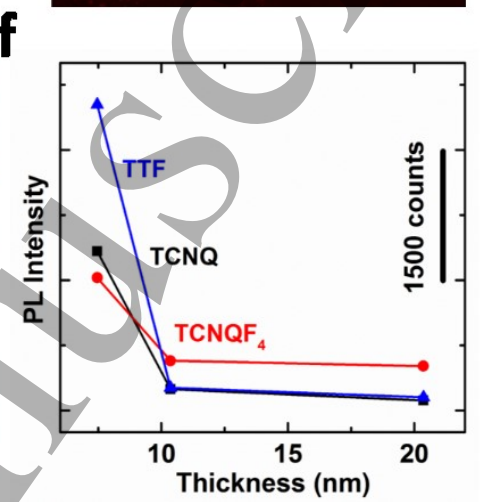

Figure S7: The AFM images of the different BP thicknesses with their thickness profiles before doping with (a) TCNQ (b,c) TCNQF 4 (d,e) TTF. The scale bar for the AFM scans are $3 \mu \mathrm{m}$ (f) The comparison of the PL intensity of 15 minutes doped (TCNQ, TCNQF $F_{4}$ and TTF) BP of thicknesses less than $10 \mathrm{~nm}, 10 \mathrm{~nm}$ and $20 \mathrm{~nm}$ are shown in the figure. 